\title{
Inverse Vaccination to Silence Immunity to Myelin in Multiple Sclerosis
}

\author{
Lawrence Steinman
}

\begin{abstract}
The adaptive immune response in multiple sclerosis is complex. We have devised large scale arrays to measure the antibody response to myelin proteins and lipids. Despite the widespread immune responses to myelin, we have devised an inverse vaccine aimed at turning off key drivers of this diverse response. Clinical trials in patients with multiple sclerosis show that it is possible to constrain antibody responses to myelin on a large scale with this approach.
\end{abstract}

\begin{abstract}
RÉSUMÉ: Vaccination inverse pour supprimer l'immunité à la myéline dans la sclérose en plaques. La réponse immunitaire d'adaptation dans la sclérose en plaques est complexe. Nous avons créé des biopuces pour mesurer la réponse immunitaire aux protéines et aux lipides de la myéline. Même si la réponse immunitaire à la myéline est largement répandue, nous avons conçu un vaccin inverse dans le but d'abolir les éléments nourriciers clés de cette réponse hétérogène. Des essais cliniques chez des patients atteints de sclérose en plaques ont montré qu'il est possible de réprimer la production d'anticorps dirigés contre la myéline sur une grande échelle en utilisant cette approche.
\end{abstract}

Can. J. Neurol. Sci. 2010; 37: Suppl. 2 - S49-S58

Eli Sercarz, one of the most creative and insightful immunologists, passed away in 2009 . He contributed greatly to our understanding of the diversity and spread of adaptive immune responses to antigens, particularly proteins. Adaptive immunity may be triggered initially in response to one component of a protein or lipid. However, this immunity no matter high tightly focused it is initially on the inciting antigen, inexorably encompasses $\mathrm{T}$ cell and antibody responses to other components of the same triggering protein, so called intramolecular spreading. The developing immune response then expands to attack components of other proteins and even lipids, often in the same anatomic vicinity ${ }^{1-3}$. Thus even in organ specific autoimmune diseases, there is a broad immunological activity directed to components of the diseased organ. In multiple sclerosis there is a diversity of $\mathrm{T}$ cell responses and antibody responses to various proteins and lipids of the myelin sheath.

The situation then becomes even more complex. Some of these 'spreading' immune responses are not only directed to constitutive components of the myelin sheath, but new immune responses target induced proteins in myelin as well as to neuronal antigens. Indeed spreading of the immune response also diversifies to recognize so called stress proteins that are induced as guardians of the tissues under attack ${ }^{4}$. Ransohoff refers to this as an "attack against the guardian" .

If one were to try to devise a vaccine that might suppress such immune responses in a disease like MS, one would have to identify the critical adaptive responses, and then to formulate a way to design what is in effect, an "inverse vaccine", one that turns off an unwanted adaptive immune response. This would in a sense be the opposite of what Jenner intended when he succeeded in vaccinating against smallpox at the end of the 18th century ${ }^{6}$. In this review I shall describe the complexity of adaptive immunity in MS, and a strategy for inverse vaccination. Results in pre-clinical models and in human clinical trials in MS, will be described with such inverse vaccines.

Sercarz wrote, "in contrast to vaccines against infectious disease, which have exploited components derived from the particular microbe or a related antigen responsible for pathogenesis, treatments for autoimmune disease have employed pharmaceutical approaches that act nonspecifically to shut off or depress general immune reactivity". We have seen in the past decade examples of powerful monoclonal antibodies like Natalizumab, that block lymphocyte migration to brain via targeting a4 integrin. Though Natalizumab has stellar efficacy in reducing relapses, approximately one per thousand individuals

\footnotetext{
From the Department of Neurological Sciences and Interdepartmental Program in Immunology, Stanford University, Stanford, California, USA.

Received April 19, 2010. Final Revisions Submitted May 26, 2010. Correspondence to: Lawrence Steinman, Department of Neurological Sciences and Interdepartmental Program in Immunology, Stanford University, Stanford, California, 94305 , USA.
} 
will develop progressive multifocal leukoencephalopathy after treatment, with the frequency increasing above this after more than two years of treatment ${ }^{7-9}$. Other approaches currently showing promise taken from the realm of cancer therapies such as anti-CD20, rituximab have also been associated with $\mathrm{PML}^{10,11}$. Other strategies such as depletion with anti-CD52, an approach successful for treatment of chronic lymphatic leukemia, have been applied to RRMS. Despite outstanding results in reducing disability and relapses in RRMS thus far, more than 40 percent of patients develop new autoimmune diseases, including thyroiditis and ITP $^{12,13}$. The need for approaches that target the adaptive immune response in MS is obvious.

\section{Complex Adaptive Immunity To Myelin Components In Multiple Sclerosis}

We developed antigen microarrays containing a spectrum of proteins and peptides derived from the myelin sheath. The 2,304feature myelin proteome arrays contain 232 distinct antigens, including proteins and sets of overlapping peptides representing MBP, PLP, MOG, myelin-associated oligodendrocytic basic protein (MBOP), oligodendrocyte-specific protein (OSP), aBcrystallin, cyclic nucleotide phosphodiesterase (CNPase) and Golli-MBP. Arrays were further customized to include key proteins and fragments thereof, including $\mathrm{aB}$ crystallin and beta amyloid, two proteins that are expressed in the injured brain and in a variety of degenerative and inflammatory conditions ${ }^{14}$. Figure 1 shows a 'heatmap' indicating some of the major antibody responses to some prominent proteins and peptides in the spinal fluid of individuals with relapsing remitting MS. In relapsing remitting MS antibodies to myelin basic protein, proteolipid protein, two heat shock proteins, hsp70 and $\mathrm{aB}$ crystallin, and beta amyloid were measured. Antibodies to $\mathrm{aB}$ crystallin were the highest titre. $\mathrm{aB}$ crystallin is a member of the family of small heat shock proteins and has neuroprotective characteristics, inhibiting apoptosis and reducing phosphorylation of NF-kB ${ }^{4}$.

In addition to the antibody responses to proteins and peptides, we have devised arrays to detect responses to some of the major lipids found in the myelin sheath ${ }^{3}$. We created ordered arrays with 50 distinct brain, myelin and microbial lipids and glycolipids that represent potential targets of the autoimmune

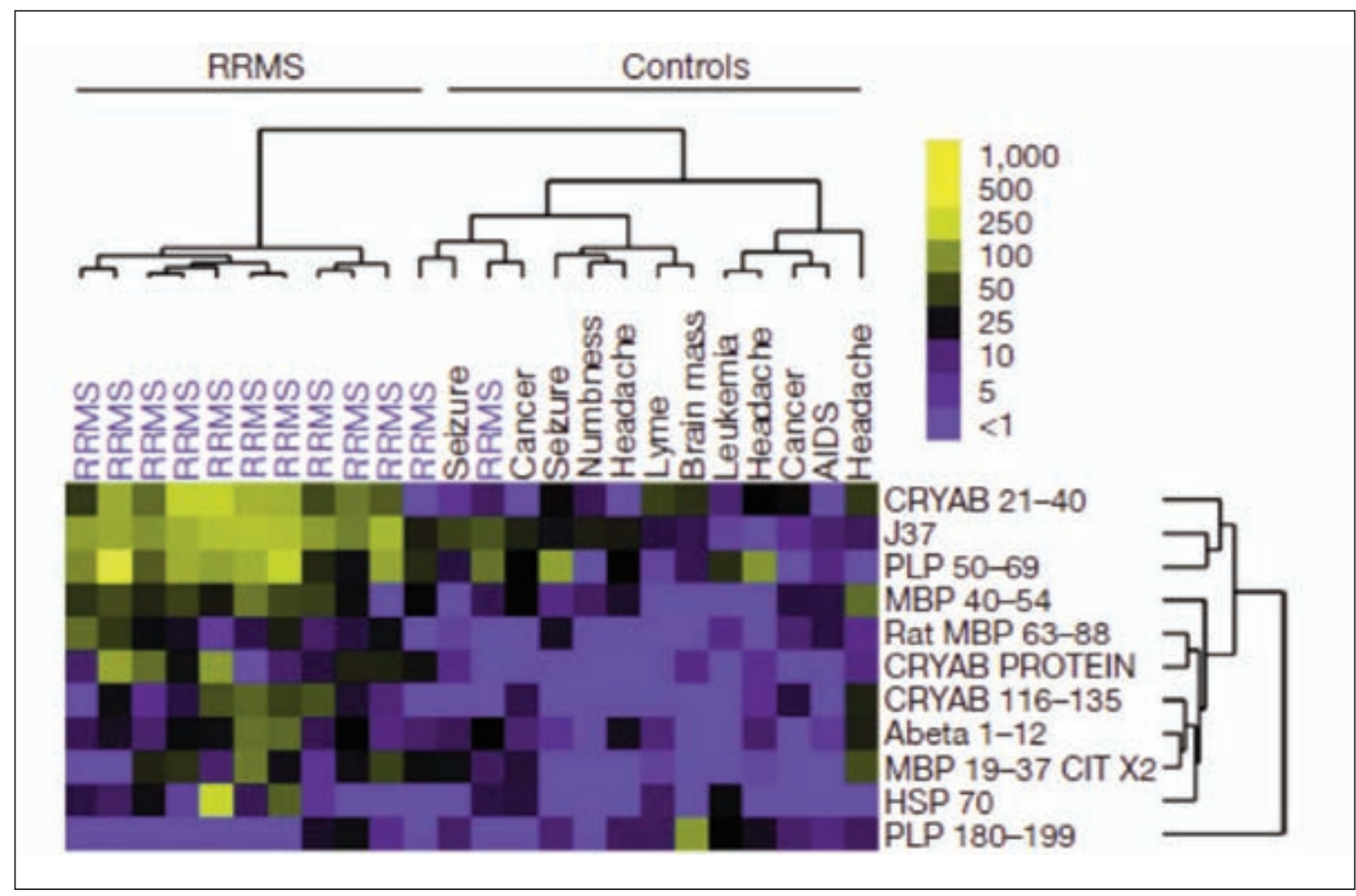

Figure 1: Permission allowed from Nature* Autoantibody responses towards proteins and peptides in the spinal fluid of patients with relapsing remitting multiple sclerosis. "Analysis was performed on CSF from RRMS and OND patients. Statistical analysis of microarrays (SAM) identified significant differences in antibodies in RRMS compared with OND. Samples are arranged with hierarchical clustering, and displayed as a heat map. RRMS patients demonstrated significantly increased auto-antibodies against various myelin epitopes including CRYAB protein and peptides (J37, Golli-myelin basic protein isoform J37); PLP, proteolipid protein; MBP, myelin basic protein; HSP, heat shock protein; Abeta, amyloid beta). A false discovery rate threshold of $1.9 \%$ and numerator threshold of 2.0 were used. Prediction analysis of microarrays (PAM) yielded a classification model with 21 markers, with cross-validated sensitivity of 10/12 = 83\% and specificity of $11 / 12=92 \%$." ${ }^{*} *$ http://www.nature.com/reprints/permission-requests.html 


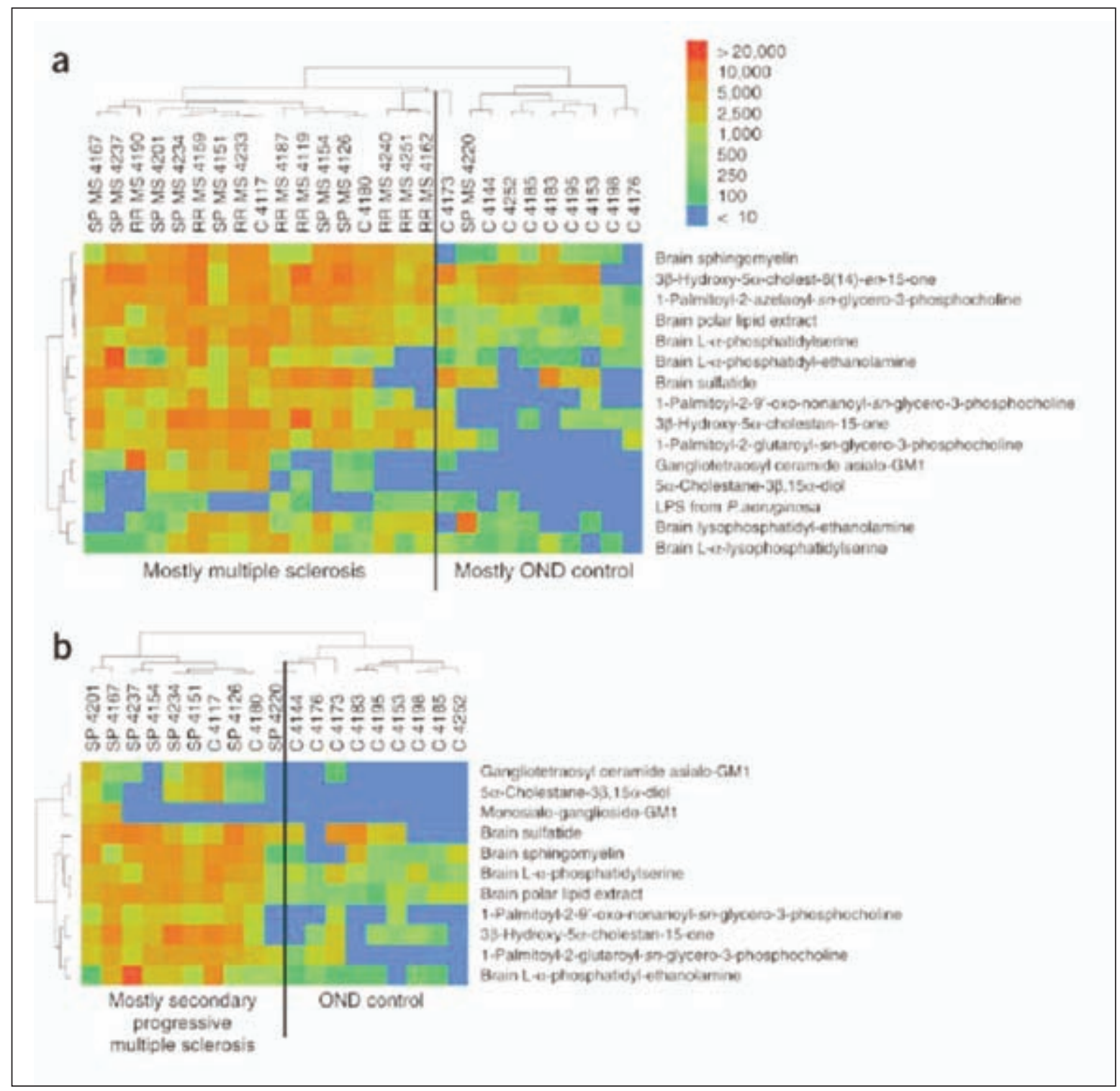

Figure 2: Permission allowed from Nature* Individuals with multiple sclerosis have increased lipid-specific antibodies. “( a) Lipid-array profiling of antibody reactivity in CSF samples from 16 subjects with multiple sclerosis and 11 OND controls. Dendrograms depicting the cluster relationships between subjects are shown above and between lipid antigens to the left. After clustering, labels were added at the base of the heatmap to indicate the general location of the clusters of individuals with multiple sclerosis and OND controls. (b) Individuals with secondary progressive multiple sclerosis possess increased ganglioside-specific antibodies compared to OND controls." 3

response in multiple sclerosis. The lipids on the arrays included ganglioside, sulfatide, cerebroside, sphingomyelin, and total brain lipid fractions, as well as microbial lipids. In a collaboration with Ingrid Catz and Ken Warren we obtained spinal fluid samples from patients with relapsing remitting and secondary progressive MS $^{3}$. We found that "The multiple sclerosis samples clustered and showed strong reactivity to lipids including sulfatide, 3-hydroxy-5-cholestan-15-one (an oxidized form of cholesterol), three separate forms of oxidized phosphatidylcholine, phosphatidyl ethanolamine, lysophosphatidyl ethanolamine and sphingomyelin, and showed weaker reactivity to bacterial lipopolysaccharide (LPS) and the ganglioside asialo-GM1. A sample from an individual "with 'other neurological disease" "who had spinal stenosis, ...clustered among the multiple sclerosis samples. When we compared only individuals with secondary progressive multiple sclerosis with the OND controls," statistical algorithms "identified increased reactivity to two gangliosides, GM1 and asialo-GM1, in the individuals with secondary progressive multiple sclerosis" Figure $2^{3}$.

Figures 1 and 2 emphasize how diverse are the targets of antibodies in various forms of multiple sclerosis. As we learn 
more about MS, other targets are likely to be discovered. Recently antibodies and $\mathrm{T}$ cell responses directed towards neuronal antigens like contactin-2 and neurofascin have been identified in $\mathrm{MS}^{15-17}$. Clearly, the adaptive immune response, most easily measured via antibodies, is very extrensive in MS.

\section{Pre-clinical Studies With Inverse Vaccines to Diverse Anti- Myelin Responses}

We tracked autoantibody responses in EAE induced with various different antigens, ranging from single peptides to homogenates of spinal cord. To do this we used the antigen microarrays containing 2,304-features with 232 distinct antigens, including proteins and sets of overlapping peptides representing
MBP, PLP, MOG, myelin-associated oligodendrocytic basic protein (MBOP), oligodendrocyte-specific protein (OSP), aBcrystallin, cyclic nucleotide phosphodiesterase (CNPase) and Golli-MBP. The complex profile autoantibody responses in serum derived from mice with EAE are presented in Figure $3^{14}$. Of note those mice showing the most diverse B-cell response also had a higher mean rate of relapses of paralysis. EAE is principally considered a $\mathrm{T}$ cell disease, so the predictive abilities of serum antibodies in these pre-clinical experiments are intriguing.

We then developed an inverse vaccine to tolerize the immune response to four constitutively expressed proteins in the myelin sheath, myelin basic protein, proteolipid protein, myelin associated glycoprotein, and myelin oligodendrocyte

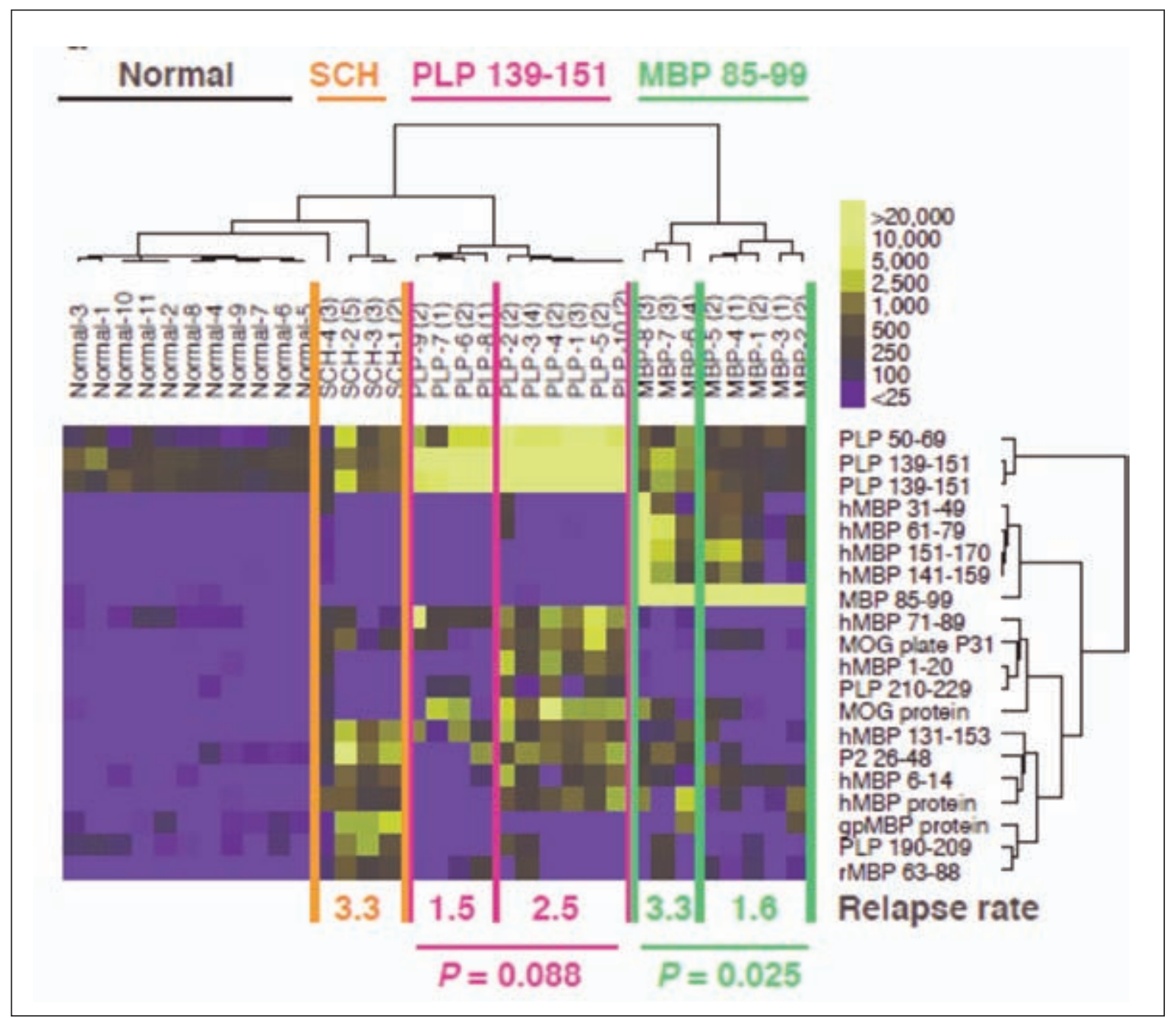

Figure 3: Permission allowed from Nature Biotechnology* "The diversity of autoantibody responses in acute EAE predicts subsequent disease activity. Hierarchical clustering of antigen features with statistically significant differences in myelin proteome array reactivity between sera derived from groups of normal control mice and from groups of mice upon recovery from acute EAE induced with PLP(139-151) (Day 17), MBP(85-99) (Day 22) or SCH (Day 25). Mice were later scored daily for ten weeks to determine the number of relapses for each mouse (indicated in parentheses). The average relapse rates for mice included in the primary subnodes of the dendrogram, and $P$ values by Mann-Whitney test for the differences in relapse rate between these nodes, are indicated" ${ }^{4}$. More epitope spreading predicts a higher relapse rate with each type of immunization for induction of EAE. 


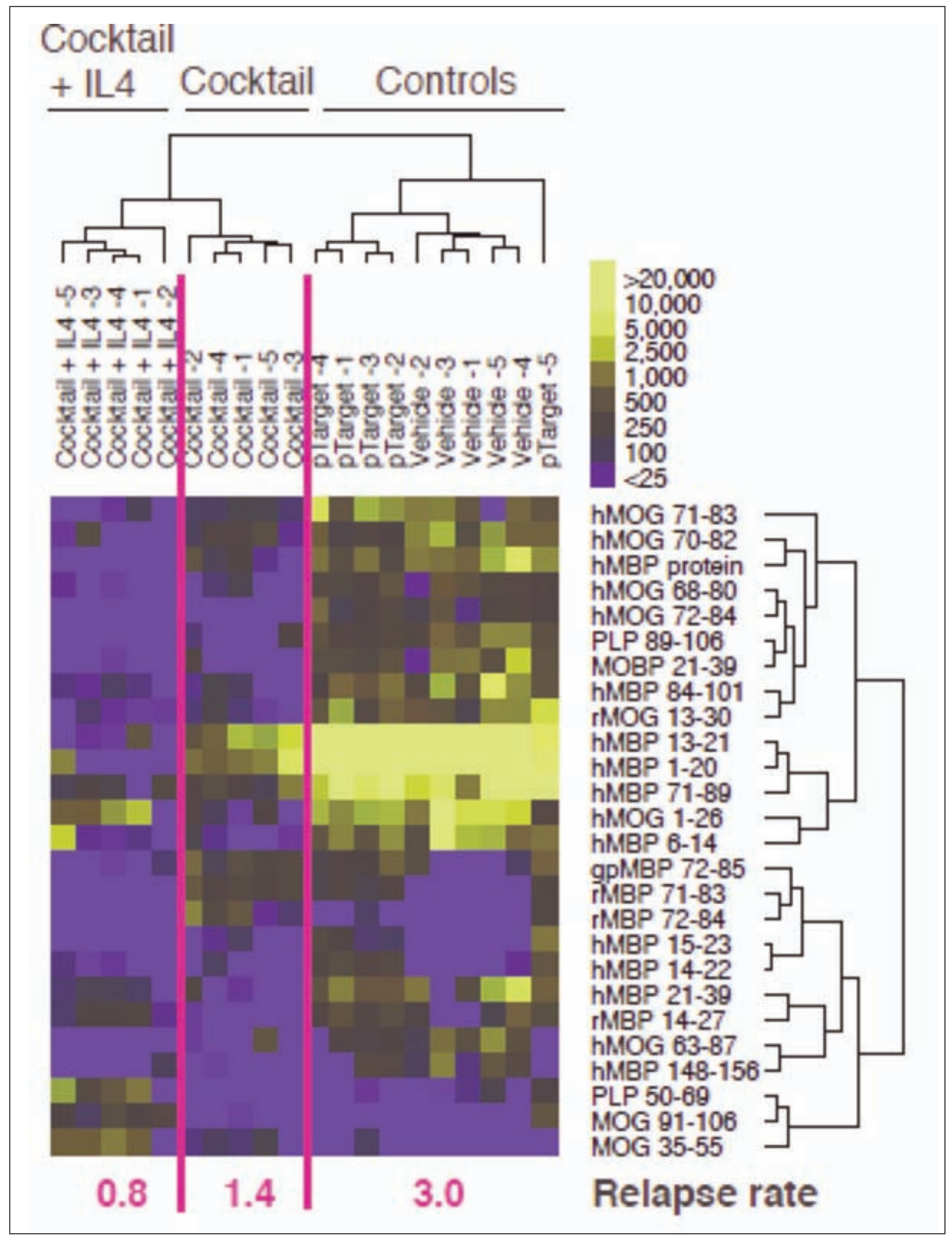

Figure 4: Permission allowed from Nature Biotechnology* "Tolerizing DNA vaccines reduce autoantibody epitope spreading. At Day 7 after onset of and after partial recovery from acute paralytic EAE (Day 17) induced with PLP(139-151), SJL/J mice were treated weekly with PBS vehicle, empty pTARGET vector, pTARGET expressing MBP, PLP, MOG and MAG (cocktail), or pTARGET expressing the cocktail and IL-4. After the ten-week treatment, serum was obtained, array analysis carried out and SAM used to identify and hierarchical cluster analysis to order antigen features." 14 


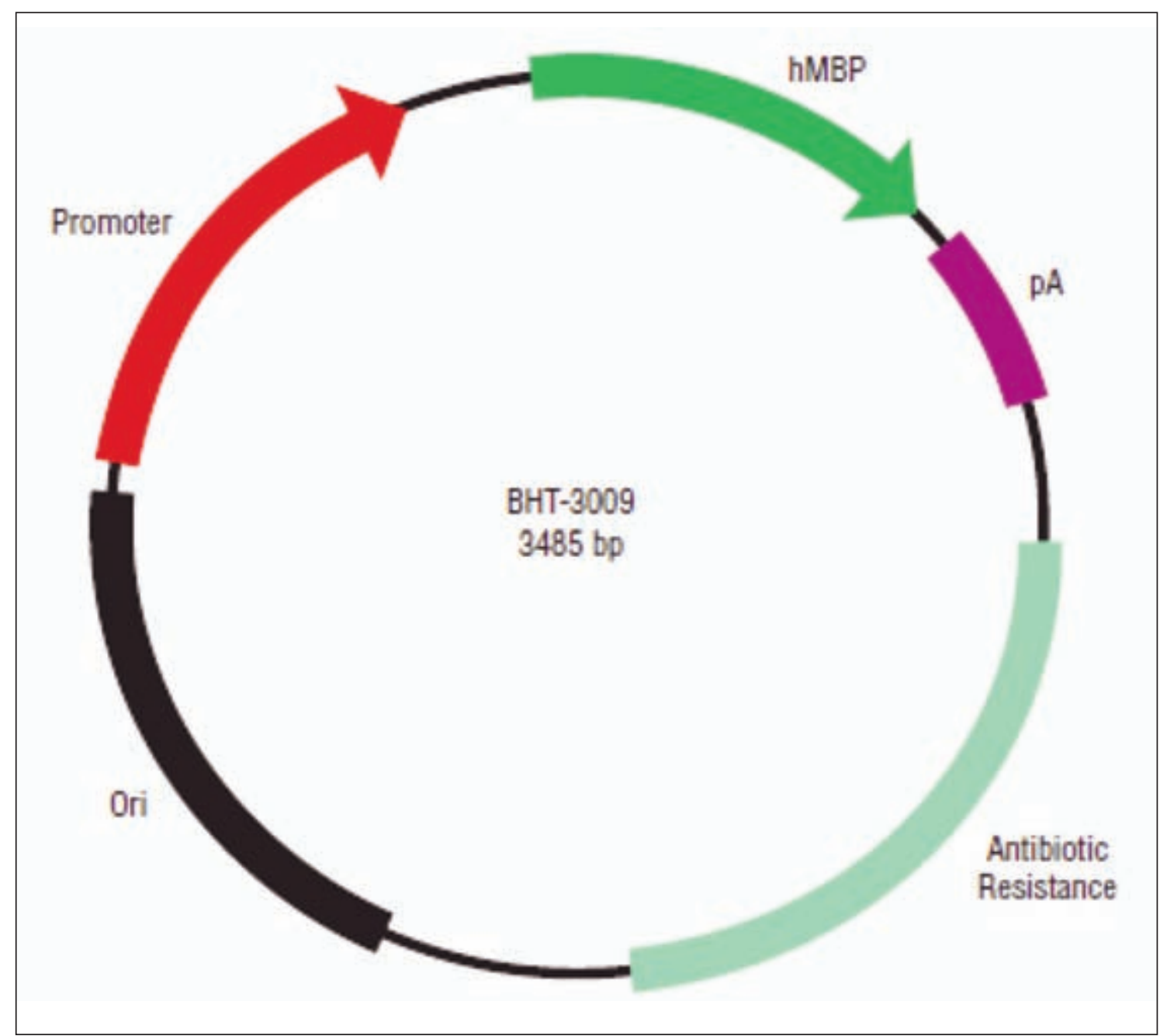

Figure 5: Permission pending from Archives of Neurology* Arch Structure of BHT-3009. The molecular structure of the 3485-base pair (bp) BHT-3009 plasmid is shown. The plasmid encodes for the 18.5-kD isoform of full-length human myelin basic protein (hMBP), which is driven by a eukaryotic promoter (promoter). Other standard elements on the plasmid include a poly A $(p A)$ region for the termination of transcription and a bacterially active origin of replication (ori) along with an antibiotic resistance gene driven by a prokaryotic promoter for propagation and manufacture of the plasmid in Escherichia coli. the full-length complementary DNA encoding the 18.5-kDa isoform of human MBP from a human brain complementary DNA library. This was cloned into a modified expression plasmid driven by a eukaryotic promoter. The expression plasmid was created as a derivative of the pVAX1 plasmid, where certain immunostimulatory CpG motifs were removed and immunoinhibitory GpG motifs were included. We have previously shown that oligonucleotides containing these GpG motifs either alone or combined with DNA vaccine plasmids were effective in treating animal models of several prototypic autoimmune diseases. The plasmid thus constructed, BHT-3009 was then manufactured under good manufacturing practice conditions and formulated in a phosphate-buffered saline solution containing calcium." 23

glycoprotein. Earlier studies with simpler versions of this strategy based on DNA vaccination, using only one plasmid encoding a PLP epitope had shown promise ${ }^{14}$. In this study "mice treated with the efficacious DNA cocktail or DNA cocktail plus IL-4 cluster showed reduced epitope spreading of autoreactive B-cell responses. In contrast, mice receiving control therapies did not discretely cluster and underwent extensive spreading of their autoreactive B-cell responses to epitopes on myelin proteins including MBP, PLP and MOG. These results demonstrate that efficacious tolerizing therapy can reduce epitope spreading of autoreactive B-cell responses." ${ }^{14}$ As is evident in Figure 3, the DNA vaccines reduced autoantibody responses to a wide spectrum of myelin proteins, including the 84-102 epitope that is one of the key drivers of demyelinating disease in certain strains of mice ${ }^{14,18,19}$, and is a key determinant of anti-myelin basic protein antibodies found in the CSF of patients with $\mathrm{MS}^{20}$. The importance of MBP 84-102 in the adaptive immune responses in MS was first described in a collaboration with Ingrid Catz and Ken Warren in $1995^{20}$. The DNA vaccines reduced relapse rates when given after the first attack of paralysis. The reduction was strong: relapses fell by approximately fifty percent with this inverse vaccine consisting 
of four plasmids encoding the major myelin antigens, and fell by over $70 \%$ when a plasmid encoding IL-4 was added ${ }^{14}$.

As mentioned there is a widespread antibody response to various lipids in MS, and in its various animal models collectively called $\mathrm{EAE}^{3}$. We have recently identified certain lipids in the myelin sheath of individuals with multiple sclerosis that provide protection from paralysis in EAE when administered to mice with ongoing disease, submitted. The lipids are anti-inflammatory and modulate key inflammatory pathways such as NF-kB. This work in progress implies that strategies can be devised to control and limit anti-lipid immunity in MS.

\section{Organ Specific Autoimmunity in Trials of Inverse Vaccines for Multiple Sclerosis}

When the work on inverse vaccination with DNA plasmids was translated from pre-clinical studies in mice to studies in man, the regulatory agencies requested for reasons of exploring

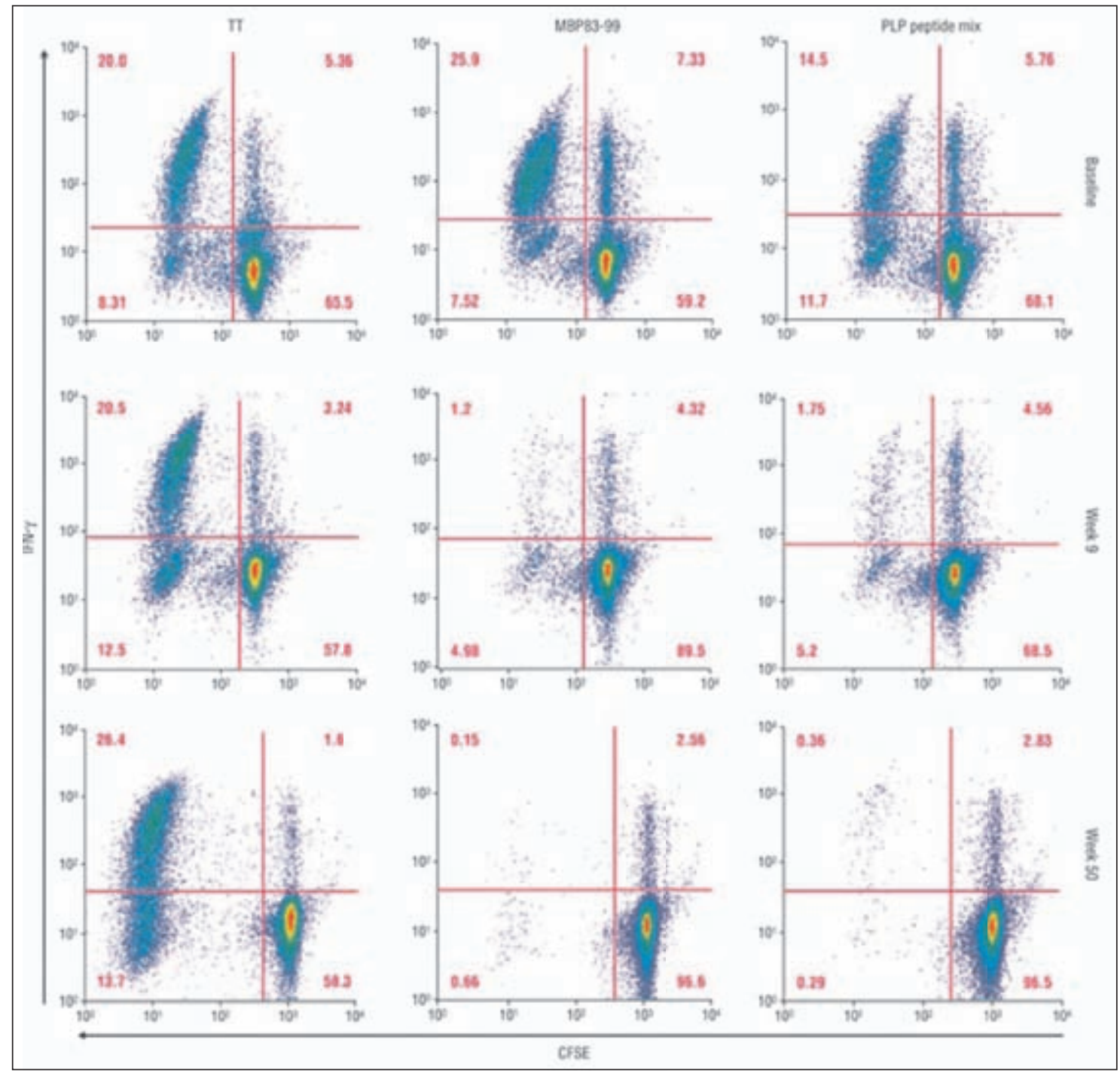

Figure 6: Permission pending from Archives of Neurology* Example of decreased T-cell response with BHT-3009. An example of one patient whose myelin basic protein (MBP) - and proteolipid protein (PLP) - specific T-cell proliferative response decreased in response to BHT-3009 is shown. Proliferation was measured using a dye dilution method with the vital dye 5,6-carboxyfluorescein diacetate succinimidyl ester (CFSE). Peripheral blood mononuclear cells were incubated with a variety of antigens and controls, but for simplicity, only the responses to tetanus toxoid (TT), MBP83-99 peptide, and a PLP peptide mix are shown. The upper three panels correspond to the baseline response; the middle three, to the Week 9 response; and the bottom three, to the Week 50 response. Proliferating interferon (IFN)--positive CD4+ T cells are shown in the upper left quadrant of each fluorescent-activated cell sorter plot. Numbers in red indicate the percentage of cells in each quadrant. A dramatic decrease in IFN-positive cells specific for MBP and PLP is demonstrated by Week 9 and persists to Week 50. Importantly, the response to TT is unchanged with dosing, confirming the antigen-specific nature of BHT-300923. 


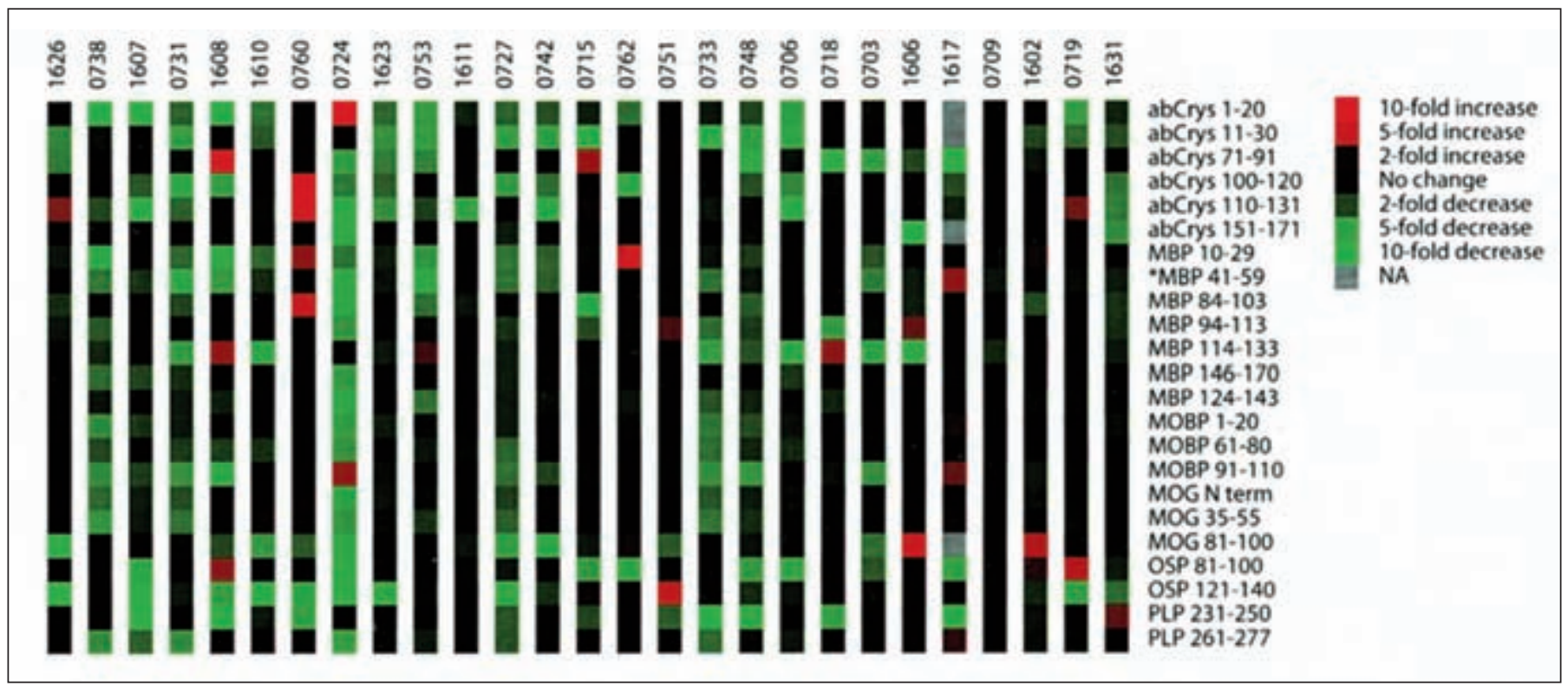

Figure 7: Permission granted from Annals of Neurology: Treatment with 0.5mg BHT-3009 is associated with a reduction in anti-myelin antibody titers. Myelin array analysis was performed to quantify anti-myelin peptide antibodies in baseline and posttreatment samples in patients treated with $0.5 m g$ BHT-3009. Antibodies with pretreatment to posttreatment changes within each treatment group are represented either as fold change in intensity with increases false colored red, no change false colored black, and decreases false colored green. Gray represents samples with no data available. Significance analysis of microarrays (SAM) was applied to identify statistical differences in antibody reactivity between the pretreatment and posttreatment samples, and identified 23 myelin peptides (listed to right of heat map) that exhibited overall significant changes (all were decreases) in the posttreatment as compared with pretreatment CSF samples in patients treated with $0.5 \mathrm{mg} B H T-3009$ (false discovery rate, $q<0.1$ ). There were no statistically significant differences between prereactivity and postreactivity in placebo-treated patients. MBP peptide sequences are derived from and numbered based on the $18.5 \mathrm{kD}$ isoform (genbank accession number AAA59562), with the exception of peptides denoted by *s which are numbered based on the $21.5 \mathrm{kD}$ isoform (genbank accession number AAA59564). MOG = myelin oligodendrocyte glycoprotein; MOBP = myelin-associated oligodendrocytic basic protein; OSP = oligodendrocyte-specific protein. ${ }^{25}$

potential safety issues, that we commence with a plasmid encoding only one myelin protein. There was always the possibility that vaccination with myelin components might worsen disease.

In order to reduce the possible pathogenicity of the inverse vaccine, many pro-inflammatory $\mathrm{CpG}$ sequences were eliminated from the plasmid backbone and replaced by an antiinflammatory sequence termed GpG, that we had discovered ${ }^{21,22}$. We had shown that a $\mathrm{GpG}$ oligonucleotide, with a single base switch from $\mathrm{CpG}$ to $\mathrm{GpG}$, inhibited the activation of Th1 T cells associated with autoimmune disease ${ }^{22}$. The $\mathrm{CpG}$ oligonucleotide with the sequence 5'-TGACTGTGAACGTTAGAGATGA-3' was modified to produce the so-called GpG oligonucleotide, 5'TGACTGTGAAGGTTAGAGATGA-3'. This immunomodulatory $\mathrm{GpG}$ oligonucleotide suppresses the severity of experimental autoimmune encephalomyelitis in mice, a prototypic immune-mediated animal disease model for multiple sclerosis. The GpG oligonucleotide stoichiometrically competes with $\mathrm{CpG}$ for binding to its receptor the toll like receptor 9, TLR9. The combination of stimulatory $\mathrm{CpG}$ oligonucleotides with inhibitory $\mathrm{GpG}$ oligonucleotides resulted in a marked reduction in phosphorylation of IKB- at $\mathrm{Ser}^{32}$. Overall, GpG oligonucleotides effectively competed with $\mathrm{CpG}$ oligonucleotides to inhibit Th1 responses ${ }^{21,22}$.
An initial clinical trial with an inverse vaccine, was attempted in thirty individuals with relapsing remitting and secondary progressive MS at four clinical sites in North America including the Montreal Neurological Institute ${ }^{23}$. Although pre-clinical testing indicated that an inverse vaccine using multiple plasmids encoding multiple myelin proteins would be optimal, after discussions with regulatory authorities in the United States and Canada, it was decided to test a vaccine, termed BHT 3009, encoding just one protein- myelin basic protein (Figure 4). BHT3009 was administered intramuscularly at Weeks 1, 3, 5, and 9 after randomization into one of three groups: placebo, BHT 3009 alone or BHT 3009 with the oral statin drug, atorvastatin. Atorvastatin was added in one group because there were indications that it may act as an adjuvant to enhance the efficacy of inverse immunization ${ }^{23,24}$.

The inverse vaccine, BHT-3009, was safe and well tolerated. There were favorable trends on brain MRI, with a reduction in gadolinium lesion activity and volume after treatment relative to the placebo group. There was no additional benefit from atorvastatin. We observed beneficial antigen-specific immune changes including a marked decrease in proliferation of interferon-gamma producing, myelin basic protein p84-102reactive $\mathrm{CD} 4+\mathrm{T}$ cells from peripheral blood (Figure 6) and a reduction in titers of myelin-specific autoantibodies from 
cerebral spinal fluid as assessed by protein microarrays ${ }^{23}$. Remarkably and unexpectedly the immune response to proteolipid protein was also reduced, though importantly the immune response to tetanus toxoid was not effected at all over a year (Figure 6) ${ }^{23}$. This was the first indication of organ specific autoimmunity. The reduction in immune responses to myelin was not only encompassing Th1 immunity to myelin basic protein, but was also reducing responses to PLP. As Sercarz might have stated it, targeting a key driver-myelin basic proteinwith an inverse vaccine, spread the suppression of immunity to other myelin proteins ${ }^{1}$. In the Phase 2 the extent of this organ specific reduction in immunity was seen at the antibody level. In the initial trial, there was also evidence at the level of antibodies: "To measure the antigen-specific immune response in the CNS as opposed to peripheral blood, patients were given the option of volunteering to undergo lumbar punctures for CSF collection. Five patients volunteered during the screening period and 3 of these patients had a follow-up lumbar puncture after completing dosing with BHT-3009. The CSF was analyzed for autoantibody titers using a protein microarray ... The results demonstrated "that treatment with BHT-3009 significantly decreased autoantibody titers in CSF to multiple myelin antigens in all three patients who had follow-up lumbar punctures. Importantly, this reduction in autoantibody titers was not restricted to MBP but was also observed with other antigens, such as proteolipid protein (PLP), suggesting that BHT-3009 can cross-tolerize to other antigenic components of the myelin sheath."23

A phase 2 trial was then performed in patients with relapsing remitting MS with the inverse vaccine, encoding myelin basic protein, termed BHT $3009^{25}$. As reported in 2008, "Compared with placebo, in the 267 patient analysis population the median four week rate of new enhancing lesions during weeks 28 to 48 was $50 \%$ lower with $0.5 \mathrm{mg}$ BHT-3009 $(\mathrm{p}=0.07)$ and during weeks 8 to 48 was $61 \%$ lower with $0.5 \mathrm{mg}$ BHT-3009 $(\mathrm{p}=0.05)$. The mean volume of enhancing lesions at week 48 was $51 \%$ lower on $0.5 \mathrm{mg}$ BHT-3009 compared with placebo $(\mathrm{p}=0.02)$. No significant improvement in magnetic resonance imaging lesion parameters was observed with $1.5 \mathrm{mg}$ BHT-3009. Dramatic reductions in 23 myelin-specific autoantibodies in the 0.5mg BHT-3009 arm were observed" in cerebrospinal fluid, "but not with placebo or 1.5mg BHT-3009."." , see Figure 7.

We had the opportunity to study a pre-defined subset of 80 patients who contributed CSF for protein array analysis at baseline, and CSF at week 44 for repeat protein array analysis ${ }^{25}$. This allowed us to determine whether treatment with BHT-3009 had an effect on the levels of anti-myelin autoantibodies. Treatment with the $0.5 \mathrm{mg}$ BHT-3009 dose was associated with a significant decrease in the autoantibody titers to 23 myelin autoantigens, whereas treatment with placebo did not result in a statistically significant net change in any of the anti-myelin autoantibodies measured in the cerebral spinal fluid. Antibody levels to MBP peptides decreased with $0.5 \mathrm{mg}$ BHT-3009. We also saw a fall in autoantibodies binding to other components of the myelin sheath in RRMS including aB-crystallin, proteolipid protein (PLP), myelin oligodendrocyte glycoprotein, myelinassociated oligodendrocytic basic protein, and oligodendrocytespecific protein (OSP). These antibodies levels to various components of the myelin sheath decreased in a statistically significant manner as determined by the SAM statistical algorithm ${ }^{25}$. This decrease in antibody responses to a wide spectrum of myelin proteins and myelin protein epitopes might indicate that once there is a decrease in the response to some of the significant drivers of autoimmunity in an organ, there is a spreading of this inverse vaccination ${ }^{25}$. This reflects in a sense the opposite of epitope spreading during conventional immunization, a phenomenon observed in autoimmune disease. Eli Sercarz and colleagues were the first to fully describe this in $\mathrm{EAE}^{1,2}$.

We studied whether baseline antibody profile in the cerebral spinal fluid predicted response to study drug. In a prospectively defined protocol we described that "in the upper half of the antiMBP reactive patients ( $\mathrm{n}=13$ on placebo, $\mathrm{n}=11$ on $0.5 \mathrm{mg}$ BHT3009 , and $n=14$ on $1.5 \mathrm{mg}$ BHT-3009), there was a significantly lower number of new Gd-enhancing lesions per patient in week 28 to 48 MRIs with $0.5 \mathrm{mg}$ BHT-3009 compared with placebo (mean \pm standard deviation, $2.5 \pm 4.03$ on $0.5 \mathrm{mg}$ BHT-3009 vs $3.3 \pm 4.59$ on placebo; $p=0.02$ ). In contrast, in this subgroup, there was no significant difference between $1.5 \mathrm{mg}$ BHT-3009 and placebo." ${ }^{25}$ Further analysis of baseline antibody profiles has allowed us to identify a serum antibody profile involving myelin basic protein where high responders had not only significant reductions in parameters of magnetic resonance imaging, but also had reductions in relapse rate in the $0.5 \mathrm{mg}$ group (in preparation). Further trials are planned in both all patients with RRMS as well as those patients pre-identified by their high responder profiles in a serum assay identifying the detailed immune response to different components of myelin basic protein.

\section{A Strategy for Inverse Vaccination to Critical Antigens that Drive Autoimmunity}

Sercarz wrote, "One relevant principle that has emerged is that once a 'driver' T-cell clone (or clones) is established, it becomes the sensible choice to target in inducing tolerance. Such a driver clone usually would recruit other TH1 clones, so controlling the driver may effectively close down the response. However, it is also likely that a dominant, small set of driver clones exists for each antigen in the target organ, so it would be an advantage to tolerize each of them. Maximum security from autoimmunity would be predicted when the crucial drivers, as well as their major recruits (to 'spread determinants'), are all tolerized." ${ }^{\prime}$ This principle was applied to studies in first animal models of MS, and now in clinical trials in MS, with some success. It is possible to attenuate antibody responses to myelin on a large scale by using inverse vaccination to the drivers of this unwanted immunity. But more work must be done, in man to make inverse vaccination a proven and accepted practice. As Sercarz concluded in his News and Views in 2003 entitled "Arraying Autoimmunity Treatment, "Further mechanistic studies to elaborate the connection between determinant spreading at the antibody level, the choice of antigens and cytokines to be used in the DNA tolerization vaccine and the true extent of reduction in the self-reactive T- (or B-) cell responses should establish the generality of these exciting results, and their usefulness in devising therapy for patient populations." 


\section{ACKNOWLEDGMENTS}

I have had a wonderful collaboration with Ingrid Catz and Ken Warren dating back to the mid 1990's when we collaborated to map the critical epitope in myelin basic protein that is a primary target and likely one of the key drivers of the immune response to myelin in MS. I salute them and cherish our collaboration. There are few in the field of MS who are working on therapies targeting the adaptive immune response in MS. Their contributions have been of immense importance to our field. Ken Warren's dedication to patient care and to research has been exemplary and represents the very concept of the compassionate physician and medical scientist.

My colleagues at Stanford University, where I have been on the faculty for three decades, have been outstanding. William Robinson, PJ Utz and Hideki Garren have been working on this every step of the way since we first met at Stanford in the late 1990's. Peggy Ho and Paulo Fontoura made seminal discoveries on the GpG motif. Colleagues at Bayhill Therapeutics particularly Nanette Solvason, Michael Leviten, Robert King, and JoAnne Quan have played critical roles in the development of DNA vaccines. None of this would have happened without support from the National Institutes of Health, the Juvenile Diabetes Research Foundation, the National Multiple Sclerosis Society and the loyal investors in Bayhill Therapeutics.

\section{CONFLICT OF INTEREST}

The author is on the Board of Directors and serves as a consultant to Bayhill Therapeutics, the developer of DNA vaccines in clinical trials for multiple sclerosis and type 1 diabetes mellitus.

\section{REFERENCES}

1. Sercarz EE. Arraying autoimmunity treatment. Nat Biotechnol. 2003;21:1017-9.

2. Lehmann PV, Forsthuber T, Miller A, et al. Spreading of T-cell autoimmunity to cryptic determinants of an autoantigen. Nature. 1992;358:155-7.

3. Kanter J, Narayana S, Ho P, et al. Lipid microarrays identify key mediators of autoimmune brain inflammation. Nat Med. 2006; $12: 138-43$

4. Ousman SS, Tomooka BH, Van Noort JM, et al. Protective and therapeutic role for aB-Crystallin in autoimmune demyelination. Nature. 2007;448:474-9.

5. Ransohoff RM. Inflammatory disease: assault against the guardian. Nature. 2007;448:421-2.

6. Jenner E. The three original publications on vaccination against smallpox by Edward Jenner. In: Harvard Classics. New York: PF Collier \& Son; 1910. vol. 38.

7. Polman $\mathrm{CH}, \mathrm{O}^{\prime}$ Connor PW, Havrdova E, et al. A randomized, placebo-controlled trial of natalizumab for relapsing multiple sclerosis. N Engl J Med. 2006;354:899-910.

8. Langer-Gould A, Atlas SW, Green AJ, et al. Progressive multifocal leukoencephalopathy in a patient treated with natalizumab. $\mathrm{N}$ Engl J Med. 2005;353:375-81.
9. Steinman L. Blocking adhesion molecules as therapy for multiple sclerosis: Natalizumab. Nature Reviews Drug Discovery. 2005; 4:510-9.

10. Hauser SL, Waubant E, Arnold DL, et al. B-cell depletion with Riutximab in relapsing-remitting multiple sclerosis. N Engl J Med. 2009;358:676-88.

11. FDA public health advisory: life-threatening brain infection in patients with systemic lupus erythematosus after Rituxan (rituximab) treatment. Rockville, MD: Food and Drug Administration, 2006. [updated 2010 January 21; cited 2008 January 18]. Available from: http://www.fda.gov/cder/drug/ advisory/rituximab.htm.

12. The CAMMS223 Trial Investigators. Alemtuzumab vs. interferon beta-1a in early multiple sclerosis. N Engl J Med. 2008;359: 1786-801.

13. Coles AJ, Wing M, Smith $\mathrm{S}$, et al. Pulsed monoclonal antibody treatment and autoimmune thyroid disease in multiple sclerosis. Lancet. 1999;354:1691-5.

14. Robinson, WH, Fontoura P, Lee BJ, et al. Reverse genomics: Protein microarrays guide tolerizing DNA vaccine treatment of autoimmune encephalomyelitis, Nat Biotechnol. 2003;21: 1033-9.

15. Steinman L. The gray aspects of white matter disease in multiple sclerosis. Proc Natl Acad Sci U S A. 2009;106:8083-4.

16. Derfuss T, Parikh K, Velhin S, et al. Contactin-2/Tag-1 directed autoimmunity is identified in multiple sclerosis and mediates gray matter pathology in animals. Proc Natl Acad Sci USA. 2009;106:8302-7.

17. Mathey EK, Derfuss T, Storch MK, et al. Neurofascin as a novel target for autoantibody-mediated axonal injury $\mathrm{J}$ Exp Med. Oct 2007;204:2363-72.

18. Sakai K, Sinha A, Mitchell DJ, et al. Involvement of distinct T cell receptors in the autoimmune encephalitogenic response to nested epitopes of myelin basic protein. Proc Natl Acad Sci USA. 1988; 85:8608-12.

19. Zamvil S, Mitchell D, Powell M, et al. Multiple discrete epitopes of the autoantigen myelin basic protein. J Exp Med. 1988;168: 1181-6.

20. Warren KG, Catz I, Steinman L. Fine specificity of the antibody response to myelin basic protein in the central nervous system in multiple sclerosis: The minimal B cell epitope and a model of its unique features. Proc Natl Acad Sci USA. 1995;92:11061-5.

21. Ho, P, Fontoura P, Ruiz P, et al. Ani GpG oligonucleotide for the treatment of autoimmunity via the innate and adaptive immune systems. J Immunol. 2003;171:4920-6.

22. Ho P, Fontoura P, Platten M, et al. A suppressive oligodeoxynucleotide enhances the efficacy of myelin cocktail/IL-4 tolerizing DNA vaccination and treats autoimmune disease. J Immunol. 2005; 175:6226-34.

23. Bar-Or A, Vollmer T, Antel J, et al. Induction of antigen-specific tolerance in multiple sclerosis after immunization with DNA encoding myelin basic protein in a randomized, placebocontrolled phase 1-2 trial. Arch Neurol. 2007;64:1407-15.

24. Youssef S, Stuve O, Patorroyo J, et al. The HMG-CoA reductase inhibitor, Atorvastatin, promotes a Th2 bias and reverses paralysis in CNS autoimmune disease. Nature. 2002;420:78-84.

25. Garren H, Robinson W, Krasulová E, et al. Phase $2 b$ trial of a DNA vaccine encoding myelin basic protein in relapsing multiple sclerosis. Ann Neurol. 2008;63(5):611-20. 